\title{
Formation of cold filaments in cooling flow clusters ${ }^{\star}$
}

\author{
Y. Revaz ${ }^{1}$, F. Combes ${ }^{1}$, and P. Salomé ${ }^{2}$ \\ 1 LERMA, Observatoire de Paris, 61 Av. de l'Observatoire, 75014 Paris, France \\ e-mail: yves.revaz@obspm.fr \\ 2 Institut of Radio Astronomy (IRAM), Domaine Universitaire, 300 rue de la piscine, 38400 Saint-Martin d'Hères, France
}

Received 24 October 2007 / Accepted 20 November 2007

\begin{abstract}
Emission-lines in the form of filamentary structures is common in bright clusters characterized by short cooling times. In the Perseus cluster, cold molecular gas, tightly linked to the $\mathrm{H} \alpha$ filaments, has been recently revealed by $\mathrm{CO}$ observations. In order to understand the origin of these filamentary structures, we have investigated the evolution of the hot ICM gas perturbed by the AGN central activity in a Perseus like cluster. Using very-high resolution TreeSPH simulations combined with a multiphase model and a model of plasma bubbles, we have been able to follow the density and temperature evolution of the disturbed ICM gas around the bubbles. Our simulations show that a fraction of the $1-2 \mathrm{keV}$ gas present at the center of clusters is trapped and entrained by the rising buoyant bubble to higher radius where the AGN heating is less efficient. The radiative cooling makes it cool in a few tens of Myr below $10^{4} \mathrm{~K}$, forming cold filamentary structures in the wake and in the rim of the bubbles. The predicted cold gas formed outside the cluster center is in agreement with the total CO mass and density profile of the observed molecular gas as well as with the kinematics of the $\mathrm{H} \alpha$ filaments. This scenario explains the $\mathrm{H} \alpha$ and $\mathrm{CO}$ filaments observed in luminous clusters without contradicting the observed lack of $1 \mathrm{keV}$ gas. It also emphasizes that if the AGN feedback provides some heating (negative feedback) it also perturbs the ICM, increasing its cooling (positive feedback).
\end{abstract}

Key words. cooling flows

\section{Introduction}

Cooling flows in clusters of galaxies has been a puzzling topic for more than 30 years (Fabian 1994; Peterson \& Fabian 2006). While early models of X-ray clusters (Cowie \& Binney 1977) applied to observations predict a large cold gas mass accretion rate, the recent data from Chandra and XMM-Newton ruled out such large amounts of cooling (Peterson et al. 2003). There are now numerous observations showing that the AGN activity may be a clue to the cooling flow problem (Fabian et al. 2000; McNamara et al. 2000; Owen et al. 2000; McNamara et al. 2000; Fabian et al. 2003a,b; Blanton et al. 2003; Wise et al. 2006; Fabian et al. 2006; McNamara \& Nulsen 2007). The emerging picture is that cooling flows are considerably reduced by the heating provided by the AGN feedback (Brüggen \& Kaiser 2001; Churazov et al. 2001; Quilis et al. 2001; Böhringer et al. 2002; Reynolds et al. 2002; Omma et al. 2004; Omma \& Binney 2004; Sijacki \& Springel 2006; Heinz et al. 2006; Cattaneo \& Teyssier 2007). The bulk of the ICM gas remains hot ( $\geq 2 \mathrm{keV})$ down to the cluster center, where it is accreted by the central black hole (Bondi accretion).

However, in this picture, it is difficult to understand the origin of the observed cold neutral gas (Edge 2001; Edge \& Frayer 2003; Salomé \& Combes 2003, 2004; Salomé et al. 2006) and the filamentary $\mathrm{H} \alpha$ structures (Conselice et al. 2001; Crawford et al. 2005). The connection between radio source and cold CO gas (Salomé \& Combes 2004) as well as the excess of optical/UV/IR light in clusters with central cooling time less than 3 Gyr are signs of the link between the filaments, the cooling and

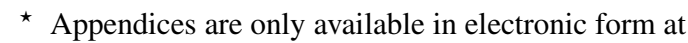
http: //www . aanda.org the AGN activity. Very deep Chandra observation of the Perseus cluster shows a clear correlation between the soft X-ray emission (between 0.5 and $1 \mathrm{keV}$ ) and the optical filamentary $\mathrm{H} \alpha$ structures (Fabian et al. 2003b, 2006). As the filaments cover a large range of cooling time within the hot gas, it is unlikely that they form by direct cooling of the $2 \mathrm{keV}$ component. Moreover, the kinematics of the filament in $\mathrm{H} \alpha$ (Hatch et al. 2006) and soft X-rays (Fabian et al. 2006) matches the gas flow under a buoyantly rising bubble. This led Fabian et al. (2003b, 2006) and Hatch et al. (2006) to propose that the filaments have been pulled out from a central reservoir of cold and warm gas by the action of radio plasma bubbles inflated by AGN jets.

In this letter, without answering the question of the inner quenching of the cooling flow, we present a series of very high resolution TreeSPH simulations of a Perseus type cluster including AGN feedback. We show that buoyantly rising bubbles entrain a fraction of the 1-2 keV central ICM gas to much higher radius where it has time to cool and fall back to the cluster center, forming cold filamentary structures $\left(T<10^{4}\right)$.

\section{The model}

\subsection{Initial mass distribution}

We have constructed a cluster model in order to fit the Perseus data from Sanders et al. (2004). The initial conditions are based on two components, a dark halo and a hot gas phase. Both share the same $\beta$-profile with $\beta=2 / 3$ (pseudo-isothermal sphere), with a core radius of $40 \mathrm{kpc}$ and a central mass density of $\rho_{0}=1.38 \times 10^{7} M_{\odot} \mathrm{kpc}^{-3}$. The gas represents $15 \%$ of the total mass and is treated as self-gravitating in the fixed potential of 
the halo. Assuming a hydrogen mass fraction of 0.76 , we found a central electron density of $0.073 \mathrm{e}^{-} / \mathrm{cm}^{3}$ and a temperature of $2.85 \times 10^{8} \mathrm{~K}$. The model is truncated at a radius of $2 \mathrm{Mpc}$, giving a total mass of $5.3 \times 10^{14} M_{\odot}$. With these parameters, the mean cooling time at the center is about $500 \mathrm{Myr}$.

\subsection{Gas physics}

Computing the interaction between the AGN and the hot ICM requires us to deal with a huge range of temperatures, spanning from more than $10^{8} \mathrm{~K}$ down to $10^{4} \mathrm{~K}$. This may be achieved by assuming a multiphase behavior of the gas where hot and cold gas represents two distinct phases.

The warm-hot gas phase $\left(T>10^{4} \mathrm{~K}\right)$ is modeled by an ideal gas with an adiabatic index of 5/3. The radiative cooling of the gas is computed from the normalized cooling function tabulated by Sutherland \& Dopita (1993), assuming an abundance of one third solar (Sanders et al. 2004). Hydrodynamical equations are integrated using the Lagrangian SPH technique.

Below $10^{4} \mathrm{~K}$, instead of quenching the cooling, the gas is assumed to change phase, becoming very cold and clumpy, as commonly observed in the ISM. In this phase, the semicollisional nature of the gas is well modeled by the sticky particle technique widely used to compute the behavior of the ISM in the disk of galaxies (Schwarz 1981; Combes \& Gerin 1985; Semelin \& Combes 2002; Bournaud \& Combes 2002).

We take into account the possibility of forming stars from the cold phase. This is computed following the standard process described in Katz et al. (1996) which simulates quite well a Schmidt law. We also take into account supernova feedback by injecting energy in both thermal (10\%) and kinetic (90\%) form, assuming that $10^{48} \mathrm{erg}$ are released for each solar mass formed.

\subsection{AGN feedback}

We have modeled the AGN feedback by instantaneously generating bubbles of gas in the ICM. This technique, which has already been used by several authors (Brüggen \& Kaiser 2001; Churazov et al. 2001; Saxton et al. 2001; Sijacki \& Springel 2006; Sijacki et al. 2007; Gardini 2007), does not consider the evolution of the jet at its origin that may be modeled by mass or energy injection (Reynolds et al. 2002; Quilis et al. 2001; Brüggen et al. 2002; Brüggen \& Kaiser 2002; Brüggen 2003; Dalla Vecchia et al. 2004; Omma \& Binney 2004; Omma et al. 2004; Heinz et al. 2006; Cattaneo \& Teyssier 2007). We have implemented a new bubble generator allowing us to control precisely the relative pressure, density and specific energy of the bubble with respect to the ambient gas. We have also taken into account the presence of the puzzling cooler and denser gas forming a bright shell around the radio bubbles (Fabian et al. 2000; McNamara et al. 2000; Blanton et al. 2001; Nulsen et al. 2002; Blanton et al. 2003; Fabian et al. 2003b, 2006; McNamara \& Nulsen 2007), asssuming that it is the result of ambient gas pushed out by the inflating bubble. From these assumptions, we can generate bubbles with densities differing from the ambient gas, without violating the total mass conservation. In a given sphere of radius $R_{\mathrm{B}}$ at a distance $D_{\mathrm{B}}$ from the cluster center, the density $\rho_{i}$, mass $m_{i}$, specific energy $u_{i}$ and pressure $P_{i}$ of a particle $i$ are modified as follows:

$$
\begin{aligned}
& \rho_{i}^{\mathrm{ICM}} \longrightarrow \rho_{i}^{\mathrm{B}}=\delta(R) \cdot \rho_{i}^{\mathrm{ICM}}, m_{i}^{\mathrm{ICM}} \longrightarrow m_{i}^{\mathrm{B}}=\delta(R) \cdot m_{i}^{\mathrm{ICM}}, \\
& u_{i}^{\mathrm{ICM}} \longrightarrow u_{i}^{\mathrm{B}}=\frac{\alpha(R)}{\delta(R)} \cdot u_{i}^{\mathrm{ICM}}, P_{i}^{\mathrm{ICM}} \longrightarrow P_{i}^{\mathrm{B}}=\alpha(R) \cdot P_{i}^{\mathrm{ICM}},
\end{aligned}
$$

Table 1. List of simulations and respective parameters. See Sect. 2 for the meaning of each parameter.

\begin{tabular}{ccccccc}
\hline \hline Id & $D_{\mathrm{B}}[\mathrm{kpc}]$ & $R_{\mathrm{B}}[\mathrm{kpc}]$ & $\alpha_{0}$ & $\delta_{0}$ & $E_{\mathrm{B}}\left[10^{60} \mathrm{erg}\right]$ & $T_{\mathrm{B}}\left[10^{7} \mathrm{~K}\right]$ \\
\hline 1 & 50 & 30 & 1.4 & 0.5 & 0.8 & 4.7 \\
2 & 50 & 30 & 1.0 & 0.5 & 0.0 & 4.1 \\
3 & 50 & 30 & 1.0 & 0.1 & 0.0 & 19.0 \\
4 & 30 & 10 & 1.0 & 0.5 & 0.0 & 4.5 \\
5 & 10 & 8 & 1.0 & 0.1 & 0.0 & 30.2 \\
\hline
\end{tabular}

where

$\delta(R)= \begin{cases}\delta_{0} & \text { if } R<R_{\mathrm{B}} \\ \delta_{0}^{\prime} \exp \left(\frac{R_{\mathrm{B}}-R}{R_{\mathrm{c}}}\right)+1 & \text { if } R \geq R_{\mathrm{B}},\end{cases}$
$\alpha(R)= \begin{cases}\alpha_{0} & \text { if } R<R_{\mathrm{B}} \\ \left(\alpha_{0}-1\right) \exp \left(\frac{R_{\mathrm{B}}-R}{R_{\mathrm{c}}}\right)+1 & \text { if } R \geq R_{\mathrm{B}},\end{cases}$

where the radius $R$ is computed with respect to the bubble center. The thickness of the rim is parametrized by $R_{\mathrm{c}}$, set to $1 / 4$ of the bubble radius. The density and pressure of the bubble with respect of the ambiant cluster gas are controlled by parameters $\delta_{0}$ and $\alpha_{0}$ (see Table 1). When $\alpha_{0}$ is set to 1 , the pressure of the bubble is not modified with respect to the ICM (see Eq. (1)). In this case, from first thermodynamic principles, no energy is injected into the system ${ }^{1}$. The constant $\delta_{0}^{\prime}$ is computed in order to conserve the total mass. According to observations, the bubble rims are slightly denser and cooler than the ambient gas, while the pressure remains unchanged.

\subsection{Simulations and parameters}

The models have been run using a modified version of the parallel TreeSPH code Gadget2 (Springel 2005) which conserves both energy and entropy. Additional physics have been included as described in paragraph 2.2 and 2.3.

In order to reach a very high resolution in the cluster central region (up to $200 \mathrm{kpc}$ ), we have constructed our model using a multi-resolution technique. The models are made out of 7 cells where two consecutive cells differ by their resolution only by a factor of two, ensuring the stability of the integration of the motion equations. This technique allows us to compute the evolution of the bubbles within a central region of $200 \mathrm{kpc}$, reaching a mass resolution of $2.4 \times 10^{6} M_{\odot}$ and a spatial resolution of about $1 \mathrm{kpc}$, appreciably better than previous works using similar Lagrangian codes. The total number of particles is 6041002 , equivalent to 33554432 particles in a similar model where no multi-resolution technique is used.

In order to explore the effect of the bubbles on the ambient gas, we have run several simulations exploring all the parameters. We have completed our study with a control run where no bubble is launched. This simulation is described in Appendix A. Hereafter, we focus on a subset of 5 representative simulations. For each simulation, the parameters are listed in Table 1 . Bubbles are created at $T=20 \mathrm{Myr}$ and are followed for $600 \mathrm{Myr}$.

Except for model 1, we have set $\alpha_{0}=1$ (bubbles are in pressure equilibrium with the ambient gas). This choice is motivated by the work of Cattaneo \& Teyssier (2007) showing that the bulk

\footnotetext{
${ }^{1}$ In fact the total energy is slightly modified, due to the particle mass and density changes which imply a change in the total potential. However, this change is very small compared to the total potential of the cluster and may be neglected.
} 
of the AGN feedback energy can be deposited at the center of the cluster in the form of thermal energy. Consequently, as we have not implemented central thermal feedback, our models are subject to the cooling catastrophe, occurring in the 10 central kpc.

\section{Results}

\subsection{Global evolution of the bubbles}

The evolution of the bubbles for the 5 models is displayed in Fig. 1. The first stage is very similar to previous works. Once generated, the bubbles quickly start moving upwards, reaching velocities of between 50 and $300 \mathrm{~km} \mathrm{~s}^{-1}$ and forming typical atomic mushrooms. However, contrary to Eulerian simulations, the latter phase does not show the formation of a torus. The bubble is only weakly affected by hydrodynamic instabilities (see Agertz et al. 2006, for a complete discussion of the damping of Kelvin-Helmoltz and Rayleigh-Taylor instabilities in SPH codes) and dissolves only when reaching regions of similar specific entropy.

\subsection{Cold gas formation}

A common feature between the 5 simulations is the systematic formation of very cold gas in the wake of the bubbles, far from the cluster core ${ }^{2}$. In a few Myrs, a substantial fraction of gas cools from the ambient ICM temperature (1-2 keV) down to $10^{6} \mathrm{~K}$, with a small fraction of it reaching temperatures below $10^{4} \mathrm{~K}$ and forming a thin trunk (see Fig. 1). The presence of cooler gas in the trunk of a buoyantly rising bubble already has been mentioned by different authors (Churazov et al. 2001; Brüggen \& Kaiser 2001; Saxton et al. 2001; Gardini 2007). However, in all these simulations, the gas temperature never falls below $5 \times 10^{6} \mathrm{~K}$. The high resolution obtained in our simulations reinforced by the Lagrangian technique enables us to resolve higher densities, critical for the correct estimation of the cooling time.

In our simulations, the filaments are formed by $1-2 \mathrm{keV}$ gas (observed in the central regions of the Perseus cluster (Fabian et al. 2006)) having short cooling time (between 400 and $800 \mathrm{Myr}$ ) at the time the bubble is formed. In the case of large bubbles (models 1-3) this gas is situated below the bubble. When smaller bubbles are created near the center (models 4,5 ), gas with sufficiently short cooling times is also found above the bubble. In both cases, this gas is trapped by the rising bubble and entrained outside the cooling cluster core where its sufficiently short cooling time allows it to cool quickly to very low temperatures. Figure 2 shows the evolution of particles becoming part of the filaments $\left(T<10^{6} \mathrm{~K}\right)$ at $t=400 \mathrm{Myr}$ for models 2 and 5 (See also Figs. B.1 and B.2 for the evolution of particles becoming part of the filaments at $t=600 \mathrm{Myr}$ ). At the beginning of simulation 2, particles being in the filaments at $t=400 \mathrm{Myr}$ (resp. $t=600 \mathrm{Myr}$ ) are part of the bottom rim of the bubble at a distance of between 20 and $40 \mathrm{kpc}$ (resp. 30 and $50 \mathrm{kpc}$ ) of the center, with a temperature between 1 and $2 \mathrm{keV}$. During the rising of the bubble, this gas is dragged up to $80 \mathrm{kpc}$ (resp. $90 \mathrm{kpc}$ ). The radiative cooling slowly decreases the gas temperature, which reaches $1 \mathrm{keV}$ around $t=300 \mathrm{Myr}$. At these temperatures, the gas is no longer supported by the pressure and falls towards the center while being compressed. Its density is enhanced by a factor of 10 which substantially decreases its

\footnotetext{
2 See Appendix A for an estimation of the cooling core in a control simulation run without feedback.
}

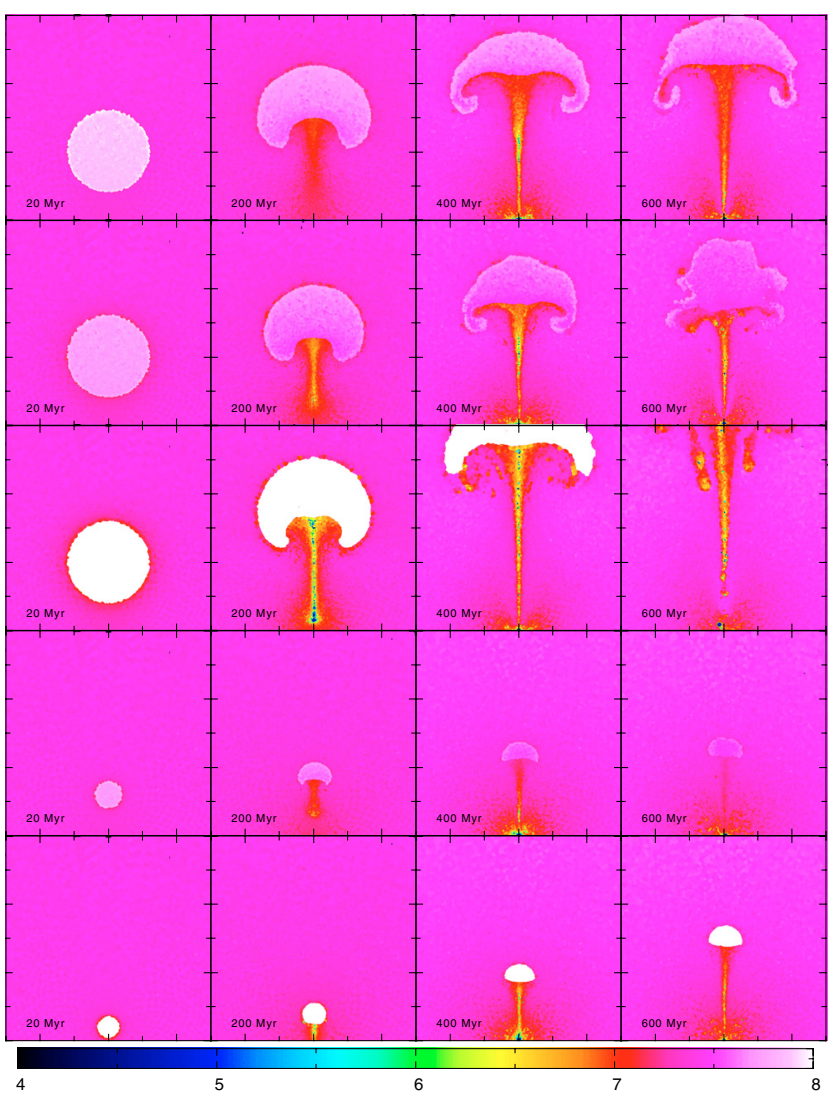

Fig. 1. Temperature map of the evolution of bubbles for models 1 (top) to 5 (bottom line). Colors represent the logarithm of the temperature between $10^{4}$ and $10^{8} \mathrm{~K}$ as indicated by the color bar. Cold gas is represented by blue colors. The temperatures are computed from a thin slice of $2 \mathrm{kpc}$ width centered on $y=0$. The width and height of each box is $150 \mathrm{kpc}$. Time increases from left to right.

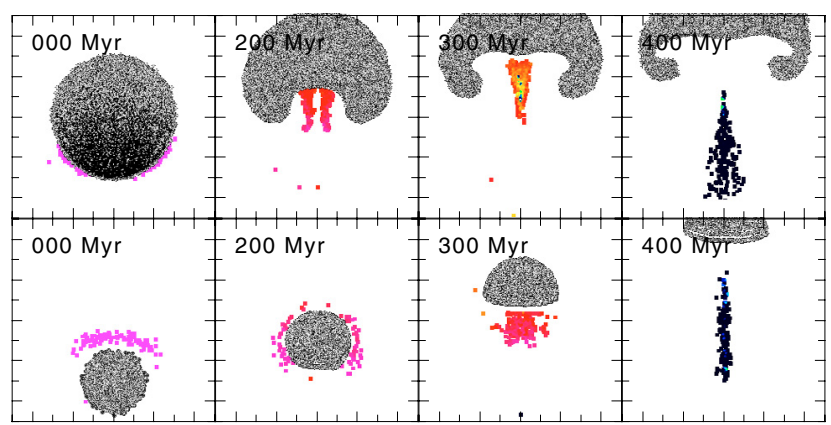

Fig. 2. Evolution of the gas around the bubble of model 2 (top) and 5 (bottom) that forms the cold filament $\left(T<10^{6} \mathrm{~K}\right)$ at $t=400 \mathrm{Myr}$. Colors have the same meaning as in Fig. 1. The plasma bubble is represented in grey. The box size is respectively $50 \times 50($ top $)$ and $25 \times$ $25 \mathrm{kpc}$ (bottom).

cooling time ${ }^{3}$. A very cold thin filament with a temperature below $10^{4} \mathrm{~K}$ is then formed. The main difference of model 5 is that the bubble is formed at smaller radius. Gas with a short enough cooling time $(<600 \mathrm{Myr})$ is initially situated above the bubble. As for the model 2, it is dragged at higher altitude (up to $60 \mathrm{kpc}$ ) and crossed by the bubble, before reaching low temperatures and forming the cold observed filament.

\footnotetext{
3 At these temperatures and densities, the cooling is so strong that it dominates the adiabatic compression heating.
} 


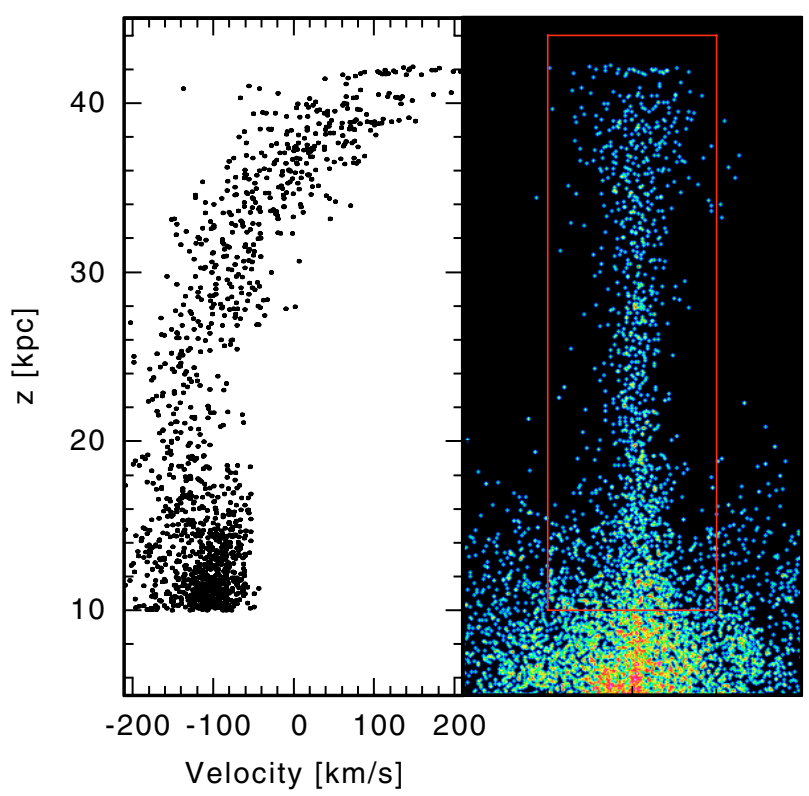

Fig. 3. Velocity of gas in the filament of model 5 at $t=400 \mathrm{Myr}$ mesured along the $z$ axis. Left: distance of the filaments from the center, as a function of their speed. Right: surface density of the gas. The particles represented in the left part of the figure are contained in the red box.

\subsection{Comparison with observations}

While a more complete model, including thermal heat, visosity and turbulence will be needed in the future, in Appendix $\mathrm{C}$ we show that the CO gas observed in the Perseus cluster may be compatible with the production of cold gas by a dozen of bubble pairs similar to bubbles of model 5 . The kinematics of the cooling gas (with temperature less than $10^{7} \mathrm{~K}$ along the filament is displayed in Fig. 3. The top of the filament is still entrained by the bubble and has positive velocities between 100 and $200 \mathrm{~km} \mathrm{~s}^{-1}$, while the bottom of the filament is falling towards the center with negative velocities. This result, showing that the filaments are stretching, is qualitatively in agreement with the observations of Hatch et al. (2006).

\section{Discussion and conclusion}

New very-high resolution SPH simulations of the Perseus cluster show that the cold gas observed in $\mathrm{H} \alpha$ and $\mathrm{CO}$ filaments outside the cluster core is a natural product of the perturbations of the ICM by buoyantly rising bubbles. During the rise of a bubble, a fraction of the low cooling time ambient ICM gas around the bubble is entrained and cools at higher radius, forming cold gas below $10^{4} \mathrm{~K}$ in the trunk of the bubble. The stretching of the filaments observed in $\mathrm{H} \alpha$ by Hatch et al. (2006) is qualitatively reproduced.

Because no central heating is taken into account, all our models are subject to the cooling catastrophe. Is the proposed scenario still valid in a model where the cooling flow is quenched by heating processes? The role of the heating source is to suppress the formation of gas with a temperature lower than $2 \mathrm{keV}$, but $1-2 \mathrm{keV}$ gas will still be present in abundance in the cluster center (Fabian et al. 2006). As seen in Sect. 3.2, this is precisely this gas that is trapped by the bubbles and later forms the cold filaments. The heating at the origin of the cooling flow quenching will thus have only a very weak influence on the amount of cold gas formed. In the new emerging picture of cooling flow,
AGN feedback provides some heating (negative feedback), but it is also responsible for a positive feedback, where ICM perturbations induce cold gas production. This is a step forward in the "cold feedback" scenario (Soker et al. 2001; Pizzolato \& Soker 2005) where the feedback takes place within a large region $(R \lesssim 5-30 \mathrm{kpc})$.

Acknowledgements. We are grateful to the anonymous referee for interesting and helpful comments. This work has been supported by the Horizon project.

\section{References}

Agertz, O., Moore, B., Stadel, J., et al. 2006, ArXiv Astrophysics e-prints Blanton, E. L., Sarazin, C. L., \& McNamara, B. R. 2003, ApJ, 585, 227 Blanton, E. L., Sarazin, C. L., McNamara, B. R., \& Wise, M. W. 2001, ApJ, 558, L15

Böhringer, H., Matsushita, K., Churazov, E., Ikebe, Y., \& Chen, Y. 2002, A\&A, 382,804

Bournaud, F., \& Combes, F. 2002, A\&A, 392, 83

Brüggen, M. 2003, ApJ, 592, 839

Brüggen, M., \& Kaiser, C. R. 2001, MNRAS, 325, 676

Brüggen, M., \& Kaiser, C. R. 2002, Nature, 418, 301

Brüggen, M., Kaiser, C. R., Churazov, E., \& Enßlin, T. A. 2002, MNRAS, 331, 545

Cattaneo, A., \& Teyssier, R. 2007, MNRAS, 376, 1547

Churazov, E., Brüggen, M., Kaiser, C. R., Böhringer, H., \& Forman, W. 2001, ApJ, 554, 261

Combes, F., \& Gerin, M. 1985, A\&A, 150, 327

Conselice, C. J., Gallagher, III, J. S., \& Wyse, R. F. G. 2001, AJ, 122, 2281

Cowie, L. L., \& Binney, J. 1977, ApJ, 215, 723

Crawford, C. S., Hatch, N. A., Fabian, A. C., \& Sanders, J. S. 2005, MNRAS, 363,216

Dalla Vecchia, C., Bower, R. G., Theuns, T., et al. 2004, MNRAS, 355, 995

Edge, A. C. 2001, MNRAS, 328, 762

Edge, A. C., \& Frayer, D. T. 2003, ApJ, 594, L13

Fabian, A. C. 1994, ARA\&A, 32, 277

Fabian, A. C., Sanders, J. S., Allen, S. W., et al. 2003a, MNRAS, 344, L43

Fabian, A. C., Sanders, J. S., Crawford, C. S., et al. 2003b, MNRAS, 344, L48

Fabian, A. C., Sanders, J. S., Ettori, S., et al. 2000, MNRAS, 318, L65

Fabian, A. C., Sanders, J. S., Taylor, G. B., et al. 2006, MNRAS, 366, 417

Gardini, A. 2007, A\&A, 464, 143

Hatch, N. A., Crawford, C. S., Johnstone, R. M., \& Fabian, A. C. 2006, MNRAS, 367,433

Heinz, S., Brüggen, M., Young, A., \& Levesque, E. 2006, MNRAS, 373, L65

Katz, N., Weinberg, D. H., \& Hernquist, L. 1996, ApJS, 105, 19

McNamara, B. R., \& Nulsen, P. E. J. 2007, ARA\&A, 45, 117

McNamara, B. R., Wise, M., Nulsen, P. E. J., et al. 2000, ApJ, 534, L135

Nipoti, C., \& Binney, J. 2004, MNRAS, 349, 1509

Nulsen, P. E. J., David, L. P., McNamara, B. R., et al. 2002, ApJ, 568, 163

Omma, H., \& Binney, J. 2004, MNRAS, 350, L13

Omma, H., Binney, J., Bryan, G., \& Slyz, A. 2004, MNRAS, 348, 1105

Owen, F. N., Eilek, J. A., \& Kassim, N. E. 2000, ApJ, 543, 611

Peterson, J. R., \& Fabian, A. C. 2006, Phys. Rep., 427, 1

Peterson, J. R., Kahn, S. M., Paerels, F. B. S., et al. 2003, ApJ, 590, 207

Pizzolato, F., \& Soker, N. 2005, ApJ, 632, 821

Quilis, V., Bower, R. G., \& Balogh, M. L. 2001, MNRAS, 328, 1091

Reynolds, C. S., Heinz, S., \& Begelman, M. C. 2002, MNRAS, 332, 271

Salomé, P., \& Combes, F. 2003, A\&A, 412, 657

Salomé, P., \& Combes, F. 2004, A\&A, 415, L1

Salomé, P., Combes, F., Edge, A. C., et al. 2006, A\&A, 454, 437

Salomé, P., Combes, F., Edge, A. C., et al. 2007, A\&A, submitted

Sanders, J. S., Fabian, A. C., Allen, S. W., \& Schmidt, R. W. 2004, MNRAS, 349,952

Saxton, C. J., Sutherland, R. S., \& Bicknell, G. V. 2001, ApJ, 563, 103

Schwarz, M. P. 1981, ApJ, 247, 77

Semelin, B., \& Combes, F. 2002, A\&A, 388, 826

Sijacki, D., \& Springel, V. 2006, MNRAS, 366, 397

Sijacki, D., Springel, V., di Matteo, T., \& Hernquist, L. 2007, MNRAS, 380, 877

Soker, N., White, III, R. E., David, L. P., \& McNamara, B. R. 2001, ApJ, 549, 832

Springel, V. 2005, MNRAS, 364, 1105

Sutherland, R. S., \& Dopita, M. A. 1993, ApJS, 88, 253

Wise, M. W., McNamara, B. R., Nulsen, P. E. J., Houck, J. C., \& David, L. P. 2006, ArXiv Astrophysics e-prints 
Y. Revaz et al.: Formation of cold filaments in cooling flow clusters, Online Material $p 1$

\section{Online Material}




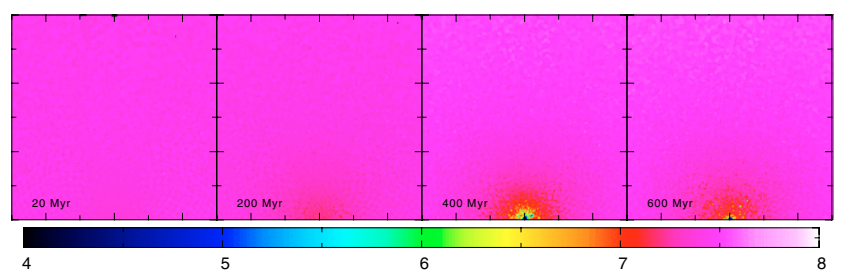

Fig. A.1. Temperature map of the evolution of the cluster for the control simulation run with no bubbles. Colors represent the logarithm of the temperature between $10^{4}$ and $10^{8} \mathrm{~K}$ as indicated by the color bar. Cold gas is then represented by blue colors. The temperatures are computed from a thin slice of $2 \mathrm{kpc}$ width centered on $y=0$. The width and height of each box is $150 \mathrm{kpc}$.

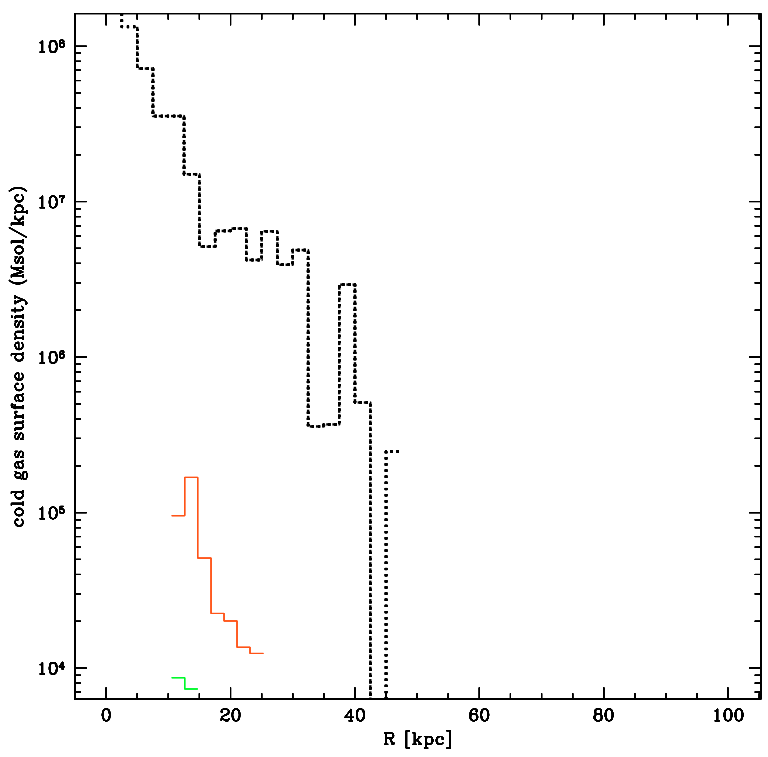

Fig. A.2. Cold gas surface density as a function of radius for the control run, at $t=400$ (green) and $t=500 \mathrm{Myr}$ (red). The dashed line corresponds to values derived from Salomé et al. (2006).

\section{Appendix A: Control run without feedback}

We present the result of the control simulation run without feedback. Figure A.1 (the equivalent of Fig. 1) shows the temperature map of the cluster. The cooling catastrophe occurs after $300 \mathrm{Myr}$. After $600 \mathrm{Myr}$, only the central $10 \mathrm{kpc}$ have temperatures below $10^{7} \mathrm{~K}$. Figure A. 3 shows the evolution of the mean temperature profile of the cluster. The cluster core is defined as the region where the temperature drops below $10^{7} \mathrm{~K}$.

Figure A. 2 shows the cold gas surface density of the outlying regions $(R>10 \mathrm{kpc})$. Those values have been used to correct the equivalent values for models with bubble perturbation presented in in Appendix C.

\section{Appendix B: Formation of cold filaments}

Figures B.1 and B. 2 show the evolution and interaction with the rising bubble of the gas of models 2 and 5, that ends in the filament at $t=600$ with a temperature below $10^{6} \mathrm{~K}$.

\section{Appendix C: Comparison with $\mathrm{CO}$ observations}

In this appendix, we compute the amount of cold gas $(T<$ $\left.10^{4} \mathrm{~K}\right)$ formed outside the cluster core $(R>10 \mathrm{kpc})$, due to the uplift of the ambient ICM gas during the bubble rise and

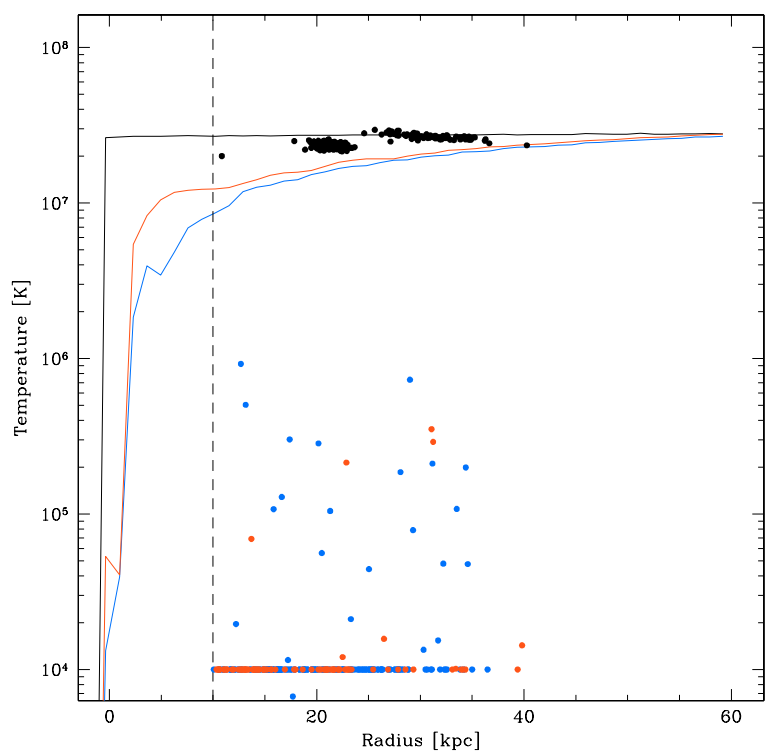

Fig. A.3. Evolution of the mean temperature profile of the reference model, corresponding to a purely cooling flow (black: $t=20 \mathrm{Myr}$, blue: $t=400 \mathrm{Myr}$, red: $t=600 \mathrm{Myr}$ ). The dashed vertical line corresponds to our definition of the core radius $\left(T<10^{7} \mathrm{~K}\right)$. Dots corresponds to the positions of particles forming a cold filament, at $t=400$ and $t=$ $600 \mathrm{Myr}$ (black: $t=20 \mathrm{Myr}$, blue: $t=400 \mathrm{Myr}$, red: $t=600 \mathrm{Myr}$, see also Fig. 2).

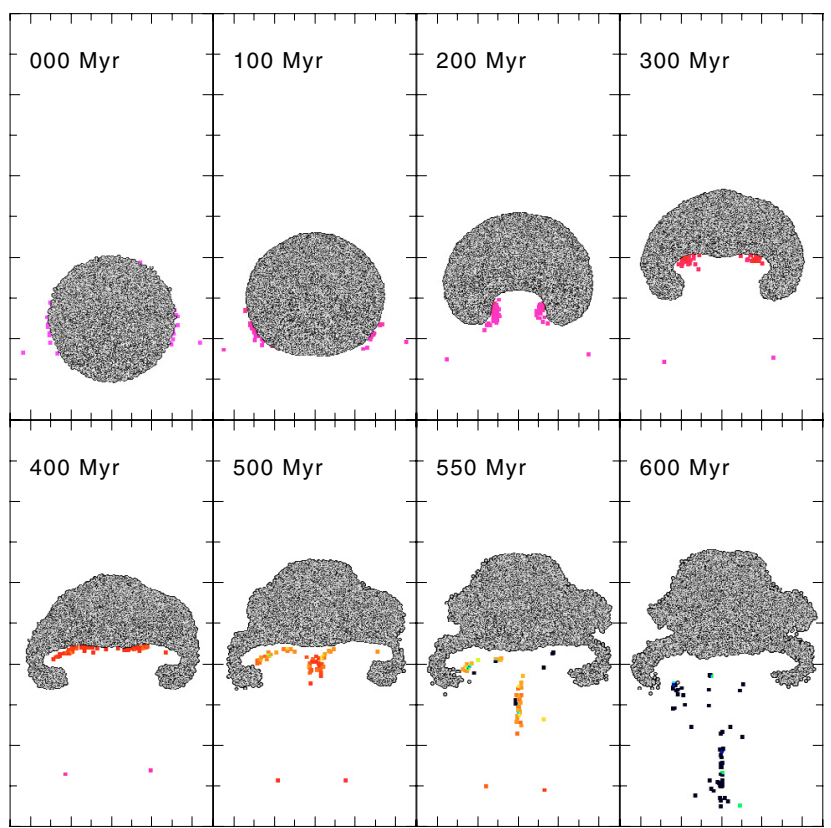

Fig. B.1. Evolution of the gas around the bubble of model 2 that form the cold filament $\left(T<10^{6} \mathrm{~K}\right)$ at $t=600 \mathrm{Myr}$. Colors represent the logarithm of the temperature between $10^{4}$ and $10^{8} \mathrm{~K}$. The plasma bubble is represented in grey. The box size is $100 \times 200 \mathrm{kpc}$.

compare it with available $\mathrm{CO}$ observation of the Perseus cluster. As no central heating is taken into account at the cluster center, a large amount of cold gas is naturally deposited within the central region (at a rate of about $160 M_{\odot} / \mathrm{yr}$ ). From the amount of gas found for each simulation, we have then subtracted the equivalent value obtained from the control simulation (see Appendix A), where no bubbles have been launched. This 
Y. Revaz et al.: Formation of cold filaments in cooling flow clusters, Online Material p 3

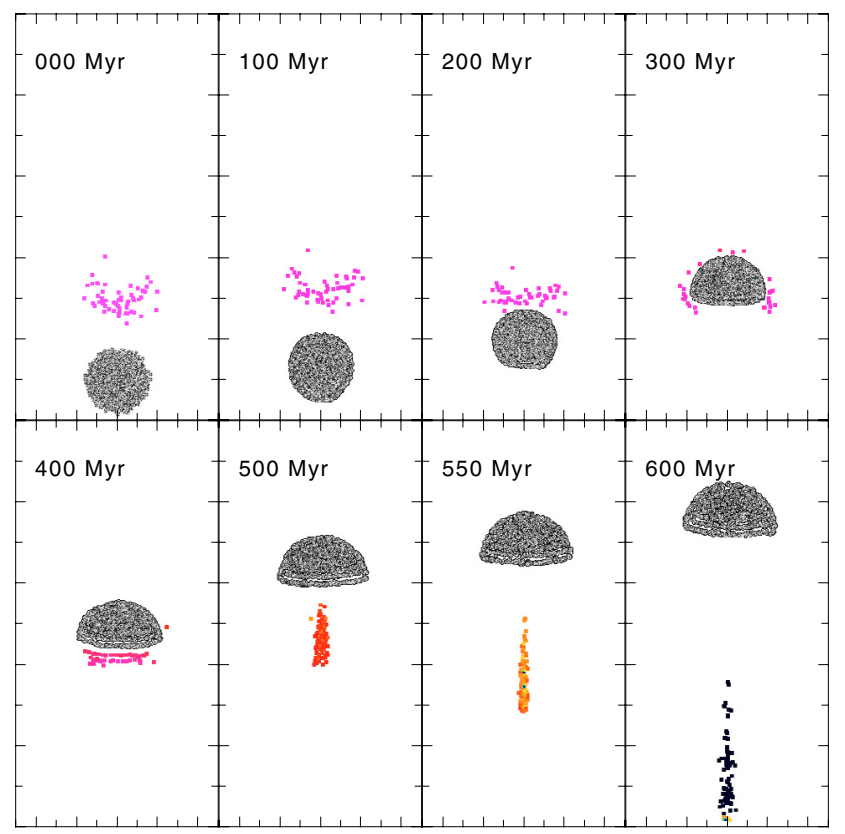

Fig. B.2. Evolution of the gas around the bubble of model 4 that form the cold filament $\left(T<10^{6} \mathrm{~K}\right)$ at $t=600 \mathrm{Myr}$. Colors represent the logarithm of the temperature between $10^{4}$ and $10^{8} \mathrm{~K}$. The plasma bubble is represented in grey. The box size is $50 \times 100 \mathrm{kpc}$.

ensures that the cold gas observed for $R>10 \mathrm{kpc}$ is only due to ICM perturbation by the rising bubbles.

Figure C.1, compares the cold gas surface density of models $1-3$ and $5^{4}$, at three different times, with the surface density of the cold gas deduced from the Salomé et al. (2006) data (dashed line). Clearly, all models fail to reproduce the amount of gas observed between 10 and $40 \mathrm{kpc}$. However, we have to recall that in those models, only two symmetric bubbles are responsible for the ICM cooling outside the cluster core. In a more complete model, taking into account the recurrent or continuous activity of the AGN will generate more bubbles, multiplying the production of cold gas by a dex. It then means that models $1-3$ probably over predict the cold gas, especially at a
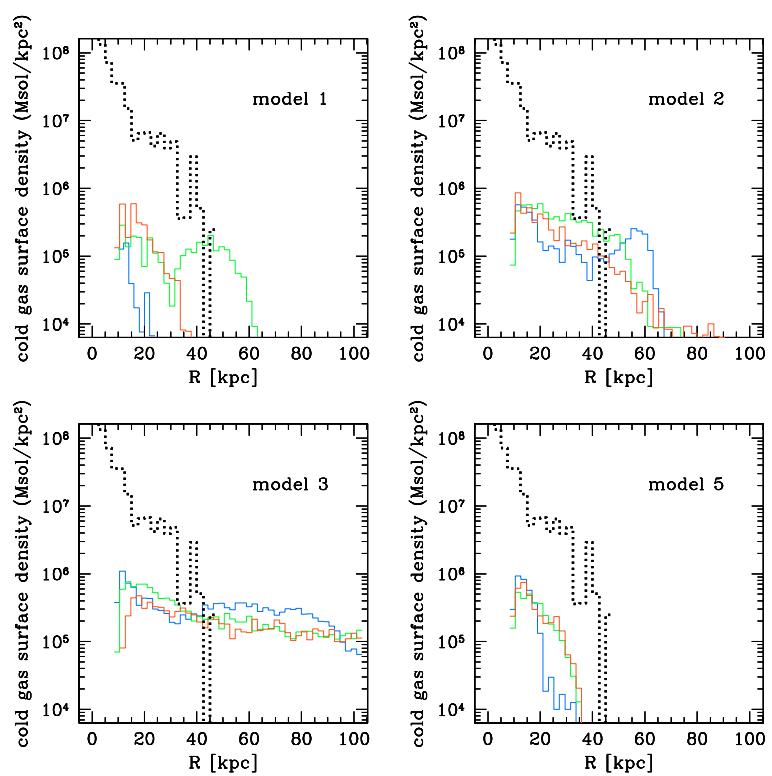

Fig. C.1. Cold gas surface density as a function of radius for models $1-3,5$, at $t=300$ (blue), $t=400$ (green) and $t=500 \mathrm{Myr}$ (red). The dashed line corresponds to values derived from Salomé et al. (2006).

radius larger than $40 \mathrm{kpc}$. However, the surface density profile of model 5 at $t=400$ and $t=500$ becomes remarkably similar to the Perseus observations. This argumentation is supported by the direct comparison of cold gas in the HorseShoe region (Pos. 11 in Salomé et al. 2007). An amount of $0.9 \times 10^{8} M_{\odot}$ has been estimated in this region, remarkably similar to the cold gas formed in the rim of the bubbles of models 2-4, ranging between 0.5 and $10 \times 10^{8} M_{\odot}$.

It is important to notice that a carefull comparison between the models and observations should also take into account the lifetime of the filaments with respect of the AGN duty cycle. Unfortunately, the lifetime of the filaments is difficult to predict because it depends on several physical processes like heat conduction (Nipoti \& Binney 2004), viscosity or turbulence, not included in the present model.

${ }^{4}$ As the cold gas production of model 4 is nearly two decades below the observations, we haven't displayed it. 